\title{
THE EVIDENCE FOR ASPECT-DEPENDENT WINDS IN Be STARS
}

\author{
Carol A. Grady \\ Astronomy Programs, Computer Sciences Corporation \\ Beltsville MD, 20708 U.S.A.
}

Karen S. Bjorkman and Theodore P. Snow

Center for Astrophysics and Space Astronomy

University of Colorado, Boulder CO 80309, U.S.A.

We present the results of a survey of stellar winds in $62 \mathrm{Be}$ and 43 normal $\mathrm{B}$ stars in spectral types $\mathrm{B} 0$ to $\mathrm{B} 5$ and luminosity classes $\mathrm{V}-\mathrm{III}$. We find that in general the wind absorption seen in the resonance lines of $\mathrm{C} \mathrm{IV} \mathrm{and} \mathrm{Si} \mathrm{IV} \mathrm{in} \mathrm{Be} \mathrm{stars} \mathrm{is} \mathrm{the} \mathrm{result} \mathrm{of}$ the superposed absorption from multiple shortward-shifted discrete absorption components. These components are observed in $63 \%$ of our Be stars, and are not observed in the normal B stars, confirming the result of Henrichs (1984).

We find evidence for a threshold in $v \sin i$ in the presence of strong and highly-ionized winds, the shape of the $\mathrm{H} \alpha$ profile, the presence of strong intrinsic linear polarization, and infrared excesses. Below $v \sin i=150 \mathrm{~km} \mathrm{~s}^{-1}$, shortward-shifted discrete components are not observed in our sample of Be stars. Above this threshhold, $77 \%$ of the Be stars in our sample have shown discrete-component absorption in one or more IUE spectra. Inspection of published $\mathrm{H} \alpha$ profiles (Dachs, et al., 1981; Andrillat and Fehrenbach, 1982; Andrillat, 1983; Barker, 1982, 1983, 1984; Peters, 1986) and written descriptions of $\mathrm{H} \alpha$ (Slettebak, 1982) indicate that double-peaked, broad or asymmetric emission profiles tend to be associated with Be stars with $v \sin i>150 \mathrm{~km} \mathrm{~s}^{-1}$. From B0.5-B4 the stars in our sample having discrete-absorption components tend also to have broad or double $\mathrm{H} \alpha$ emission. Waters (1986) has observed a threshold in $12 \mu \mathrm{m}$ IR color excess in Be stars at approximately $v \sin i=200 \mathrm{~km} \mathrm{~s}^{-1}$. McLean and Brown (1978) have reported a threshold for the detection of significant optical polarization at $v \sin i=150 \mathrm{~km} \mathrm{~s}^{-1}$.

The change in the observed characteristics of Be stars at $v \sin i=150 \mathrm{~km} \mathrm{~s}^{-1}$ suggests that the same underlying mechanism causes the infrared excesses, the linear polarization, the $\mathrm{H} \alpha$ emission profile shapes, as well as the highly-ionized stellar winds in these stars. Recent studies have suggested that non-radial pulsations are common in Be stars. The strongest arguments favoring a pulsational origin for the Be-phenomenon are that the pulsational modes change at approximately $v \sin i=150 \mathrm{~km} \mathrm{~s}^{-1}$ (Smith and Penrod, 1985) and that the time scales for the strong wind episodes are similar to time scales for the development and dissipation of characteristic pulsational modes (Grady, et al., 1986; Smith and Penrod, 1985).

The available polarimetric and infrared data suggest that the outer atmospheres of Be stars are not spherically symmetric. If we assume that the Be stars are a population of rapidly rotating stars viewed at random inclinations, the low $v \sin i$ stars can be interpreted as being viewed at higher latitudes than higher $v \sin i$ objects. The absence of shortward-shifted discrete components below $v \sin i=150 \mathrm{~km} \mathrm{~s}^{-1}$ suggests that the highly-ionized wind does not extend to polar latitudes. In two intermediate $v$ sin $i$ stars in our sample, contemporary linear polarization and UV observations have shown that the wind variability and the linear 
polarization changes are apparently uncorrelated (Sonneborn, et al., 1986; Grady, et al., 1986), suggesting that the highly-ionized stellar wind is separated in latitude from the presumably equatorial disk responsible for the linear polarization. Previous surveys of $\mathrm{Be}$ stars have noted that emission in the UV resonance lines is comparatively rare (Barker et al., 1984). Only a few stars in our sample show suggestions of emission in C IV, and these objects tend to be those known to show strong optical shell spectra. If the shell stars are viewed close to equator-on, the presence of emission in C IV and sometimes N V suggests that a substantial fraction of the highly-ionized stellar wind occurs above the equatorial plane. These stars also tend to show only weak low-velocity absorption in C IV and Si IV, further suggesting that the bulk of the highly-ionized material is not in the line of sight.

\section{REFERENCES}

Andrillat, Y., 1983, Astron. Astrophys. Supp. Ser., 58, 319.

Andrillat, Y. and Fehrenbach, Ch., 1982, Astron. Astrophys. Supp. Ser., 48, 93.

Barker, P.K., 1982, Be Star Newsletter, 6, 34.

Barker, P.K., 1983, Be Star Newsletter, 7, 10.

Barker, P.K., 1984, Be Star Newsletter, 9, 20.

Barker, P.K., Marlborough, J.M., and Landstreet, J.D., 1984, in The Future of Ultraviolet

Astronomy: Six Years of IUE Research, eds. Mead, J.M., Chapman, R.D., and Kondo, Y., NASA CP-2349, p. 219.

Dachs, J., et al., 1981, Astron. Astrophys. Supp. Ser., $4 \$, 427$.

Grady, C.A., Bjorkman, K.S., and Snow, T.P., 1986, Ap.J. (submitted).

Henrichs, H.F., 1984, in Proceedings of the Fourth European IUE Conference, Roma, ed.

Rolfe, E. and Battrick, ESA SP-218, p. 43.

McLean, I.S. and Brown, J.C., 1978, Astron. Astrophys., 69, 291.

Peters, G.J., 1986, (private communication).

Slettebak, A., 1982, Ap.J. Supp., 50, 55.

Smith, M.A., and Penrod, G.D., 1985, in Relations Between Chromospheric-Coronal Heat-

ing and Mass-Loss in Stars, Trieste Workshop Series, 1984: Sunspot, New

Mexico, eds. Stalio, R., and Zirker, J., p. 394.

Sonneborn, G., et al., 1986, Ap.J. (submitted).

Waters, L.B.F.M., 1986, Astron. and Astrophys Lett. (in press). 


\section{DISCUSSION FOLLOWING GRADY}

Friend:

In your plot of CIV edge velocity normalized to escape velocity, are you using the escape velocity at the photosphere?

Grady:

Yes.

Friend:

Presumably, the CIV edge velocity refers to a point somewhere out in the wind where the local escape velocity is much smaller than the photospheric value. If this is the case, then there is no problem moving the material to infinity.

Grady:

This is correct, but without a detailed model it is impossible to estimate what velocities are reached at any given radius, other than at the photosphere.

Waters:

Concerning the relation between the existence of narrow components and vsini, the IRAS data show that B-type dwarfs generally show no IR excess if vsini is less than $100-200$ $\mathrm{km} / \mathrm{s}$, whereas the stars can show large excesses at $12 \mu$ if they have vsini $>100-200$ $\mathrm{km} / \mathrm{s}$.

Grady:

I think the most exciting finding of our survey is the threshold for strong and variable winds dominated by discrete components at vsini $=150 \mathrm{kms}^{-1}$, and the fact that similar thresholds are seen in the optical polarization, Balmer line profiles, FeII emission profile, infrared excess, and non-radial pulsation data. It suggests that the Be outer atmosphere, in all of its manifestations, may be controlled by the same mechanism.

Furenlid:

Evidence for mass flow in $\mathrm{H} \alpha$ for normal B stars in the range BO-B3 can be seen only when vsini - and thereby $\mathrm{v}$ - is larger than $200 \mathrm{kms}^{-1}$. This threshold velocity increases through the $B$ stars and reaches around $300 \mathrm{kms}^{-1}$ at $\mathrm{AO}$.

Grady:

We have suggestions of a threshold for CIV and SiIV as a function of spectral type in our sample of Be stars. In particular, we observe no luminosity class $V$ or IV Be stars with CIV and SiIV. Our sample contains relatively few observations of B4 and B5e stars, so our results can only be considered preliminary, and should be checked when analysis of a sample of late-type Be stars has been completed. 